\title{
Online Attitude Determination of a Passively Magnetically Stabilized Spacecraft
}

\author{
R. Burton ${ }^{\mathrm{a}, \mathrm{b}, \mathrm{c}, *}$, S. Rock ${ }^{\mathrm{a}}$, J. Springmann ${ }^{\mathrm{d}}$, J. Cutler $^{\mathrm{d}}$ \\ ${ }^{a}$ Stanford University, 496 Lomita Mall, Stanford, CA 94305, United States \\ ${ }^{b}$ NASA Ames Research Center, Moffett Field, CA 94035, United States \\ ${ }^{c}$ Millennium Engineering and Integration Services, 2231 Crystal Dr, Arlington, VA \\ 22202, United States \\ ${ }^{d}$ University of Michigan, 1320 Beal Ave, Ann Arbor, MI 48109, United States
}

\begin{abstract}
An online attitude determination filter is developed for a nano satellite that has no onboard attitude sensors or gyros. Specifically, the attitude of NASA Ames Research Center's O/OREOS, a passively magnetically stabilized 3U CubeSat, is determined using only an estimate of the solar vector obtained from solar panel currents. The filter is based upon the existing multiplicative extended Kalman filter (MEKF) but instead of relying on gyros to drive the motion model, the filter instead incorporates a model of the spacecraft's attitude dynamics in the motion model. An attitude determination accuracy of five degrees is demonstrated, a performance verified using flight data from the University of Michigan's RAX-1. Although the filter was designed for the specific problem of a satellite without gyros or attitude determination it could also be used to provide smoothing of noisy gyro signals or to provide a backup in the event of gyro failures.
\end{abstract}

Keywords: Attitude determination, Nano satellites, Passive magnetic stabilization, Kalman Filter, MEKF, Online estimation

\section{Introduction}

In this paper the problem of providing an online attitude determination capability to satellites that have no dedicated attitude or attitude rate sens-

\footnotetext{
${ }^{*}$ Corresponding author

Email address: rolandb@alumni.stanford.edu (R. Burton)
} 
ing hardware is studied. The specific problem studied originated from work 5 relating to NASA Ames Research Center's O/OREOS[1] nano satellite, a 3U CubeSat illustrated in Fig. 1(a). The primary science mission on O/OREOS required neither an attitude pointing nor an attitude knowledge capability. To minimize mission cost, complexity and risk, a passive magnetic attitude stabilization system was used and no attitude or attitude rate sensing hardware was installed. After launch and completion of the primary science mission, however, there was a subsequent desire to estimate the attitude of the spacecraft to help with the design of a future mission. The only data available to perform this task were the electrical currents from the body mounted solar panels from which an estimate of the body frame sun vector, the unit vector pointing from the spacecraft to the sun, can be made.

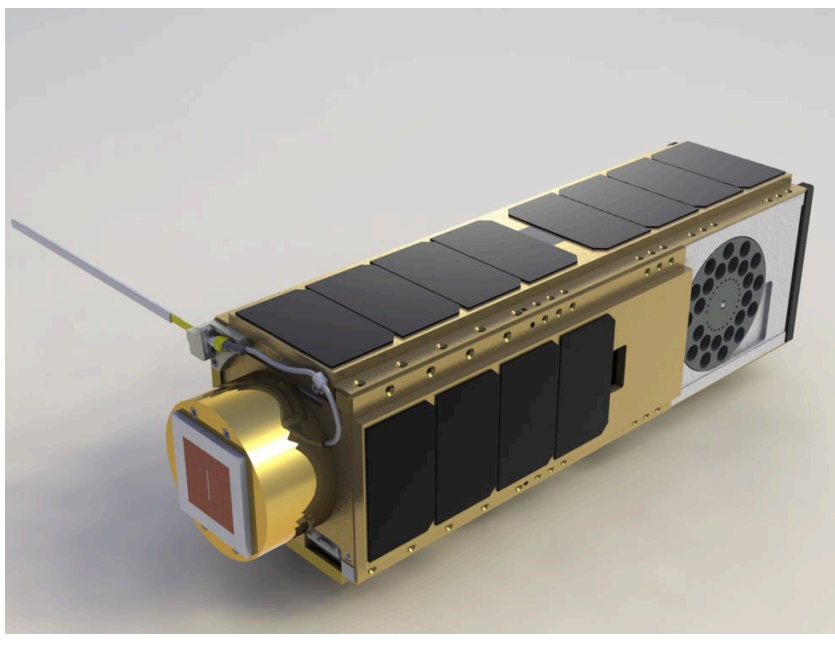

(a) O/OREOS (NASA)

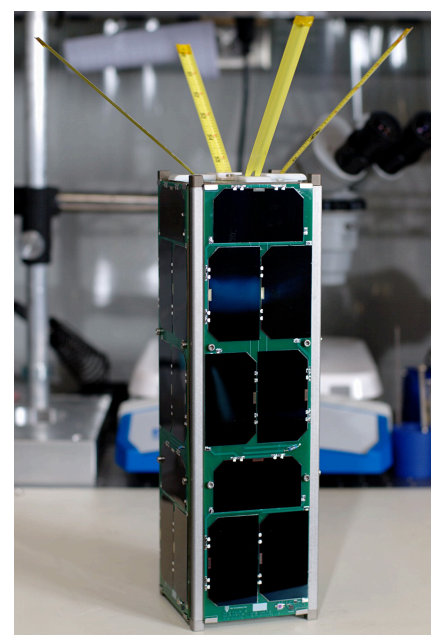

(b) RAX-1 (University of Michigan)

Figure 1: 3U CubeSat nano satellites

A single isolated measurement of a known inertial vector, such as the sun vector, is insufficient to determine attitude due to a rotational ambiguity around the vector itself. Taking sequential measurements of the vector can help, although if the spacecraft is undergoing torque free motion then the ambiguity will still remain. To resolve the ambiguity, the spacecraft's attitude motion either needs to be forced with a known external torque that is aperiodic with the rotation in the body frame, or the known inertial vector being measured needs to be moving in the inertial frame. For O/OREOS, the 
passive magnetic stabilization system provides the external torques necessary to resolve the ambiguity.

Attitude estimators that use sequential measurements incorporate a motion model whose purpose is to propagate the estimate between each measurement. Existing attitude determination algorithms that incorporate a motion model[2, 3], have relied on gyro readings to drive the motion model and so are not suitable for use in this case, where the spacecraft does not have gyros. The online attitude filter presented in this paper instead uses a model of the spacecraft's attitude dynamics as the motion model, allowing attitude determination to be performed using only sequential sun vector measurements and no onboard gyros.

35 The new filter is based upon the popular multiplicative extended Kalman filter [4] (MEKF), a recursive estimator that in its original formulation uses a gyro driven motion model. In an MEKF, spacecraft attitude is represented by the unit quaternion. In this work, the gyro-driven kinematic motion model in the original MEKF is replaced with a model of the spacecraft attitude dynamics, a two step process requiring the formulation of an attitude dynamics model and the reformulation of the original filter equations to account for the different motion model.

Formulating an accurate attitude dynamics model is non-trivial due to the large uncertainties in inertia properties and external torques [3]. Recent work [5], however, has shown that an attitude dynamics model can be reconstructed using a batch parameter estimation process. Reformulating the MEKF to incorporate an attitude dynamics model is presented in this paper.

While in this paper the new filter is only applied to the specific case of a passively magnetically stabilized nano satellite, the filter is applicable to 50 any spacecraft for which there exists (1) an accurate dynamics model (2)the ability to measure an inertial vector and (3) either known external torques or movement of that inertial vector to resolve the rotational ambiguity.

Although the motivating problem concerns a satellite without any attitude sensors, the new filter does also have utility in modern spacecraft designs that do include dedicated attitude sensors. Firstly, the algorithm can provide a backup attitude determination capability in the event that some or all of the dedicated attitude or attitude rate sensors fail or for use when the spacecraft is in safe-mode and not all systems are operational. Secondly, the gyro-free MEKF can be trivially modified to include both gyro measurements and the attitude dynamics model. This has the potential to provide an improved attitude determination capability over just using gyros when the 
gyros are noisy, as is often the case with the MEMS gyros now commonly used on nano satellites.

The new filter is tested and shown to converge using actual flight data from O/OREOS. Unfortunately, as O/OREOS contained no attitude hardware, no independent estimate of attitude is available and filter performance cannot be verified. In order to verify filter performance, the filter is also applied to flight data from the University of Michigan's RAX-1 [6], illustrated in Fig. 1(b), Like O/OREOS, RAX-1 was a passively magnetically stabilized 3U CubeSat. Unlike O/OREOS, however, RAX-1 also carried attitude sensors, including a gyro, magnetometers and photodiode sun sensors. An independent attitude estimate is made using traditional methods from these sensors and compared to the estimate generated using only measurements of the sun vector and a model of the satellite's attitude dynamics. Attitude determination performance of $5^{\circ}$ with the new filter is observed.

This paper is organized as follows: Section 2 describes the nano satellites studied in this paper and details the flight data available from each. A gyro free MEKF, where the motion model has been changed from the customary one utilizing gyros to one based on an attitude dynamics model is presented in Section 3. In Section 4 the new gyro-free filter is implemented for a passively magnetically stabilized nano satellite and that section includes the derivation of the required attitude dynamics model. Results from testing the filter in simulation are presented in Section 5 and results from application to actual flight data are presented in Section 6 .

\section{The Spacecraft}

Passive magnetic stabilization is commonly employed in nano satellites where a precision pointing capability is not required. The spin axis of a passively magnetically stabilized spacecraft stays nominally aligned with the local magnetic field vector, providing rudimentary nadir pointing. The low mass and zero power requirements of the system make its use ideal in nano satellites. The two satellites considered in this paper, NASA Ames Research Center's O/OREOS and the University of Michigan's RAX-1, are both passively magnetically stabilized $3 \mathrm{U}$ CubeSats. The passive magnetic stabilization system provides the external torques that are required to resolve the rotational ambiguity around the single measurement vector and allow attitude determination to be performed. 


\subsection{Spacecraft Background}

The Organism/Organic Exposure to Orbital Stresses (O/OREOS) spacecraft, illustrated in Figure 1(a), was a 3U CubeSat that carried two astrobiology payloads to study the survivability and viability of the space environment to live organisms and organics respectively. The O/OREOS spacecraft's passive attitude stabilization system consisted of permanent dipoles along the long axis, and hysteresis rods in the plane perpendicular to the long axis. While O/OREOS had no direct onboard attitude sensing, the spacecraft bus did monitor solar panel currents.

The first Radio Aurora Explorer satellite, RAX-1, another 3U CubeSat illustrated in Figure 1(b), was developed to study magnetic field-aligned plasma irregularities in Earth's ionosphere. The satellite was developed jointly by SRI International and the University of Michigan. The science payload was an ultra high frequency (UHF) radar receiver. Working in conjunction with ground based incoherent scatter radar stations, the purpose of the mission was to improve the understanding of the ionospheric irregularities with the ultimate goal of enabling short-term forecasting. The passive magnetic attitude control system consisted of four permanent magnets aligned with the long $z$ axis and two strips of HyMu80 soft magnetic material mounted in two axes perpendicular to the permanent magnets. RAX-1 included a full suite of attitude sensors consisting of multiple photodiodes, two three-axis magnetometers, and a three-axis rate gyroscope[7]. To improve the accuracy of the magnetometer and photodiode measurements, an attitudeindependent calibration was performed using an on-orbit magnetometer calibration algorithm developed to mitigate the effect of nearby electronics on the magnetometers, which are embedded in the spacecraft 8 ].

\subsection{Spacecraft Data}

Both satellites were secondary payloads on the same November 2010 launch from Kodiak AK, and were inserted into a $650 \mathrm{~km}$ altitude, $72^{\circ}$ inclination low Earth orbit. After separation from the launcher, orbital position was determined by propagating two-line-element (TLE) ephemerides using the SGP4[9] propagator. TLEs were updated daily, leading to maximum orbit propagation errors of a few kilometers.

Both O/OREOS and RAX-1 recorded onboard data at $1 \mathrm{~Hz}$ during data capture windows. Several data capture windows were available for the O/OREOS spacecraft and each window was typically a few minutes long. For the RAX-1 spacecraft, three data capture windows were available, and each was around 


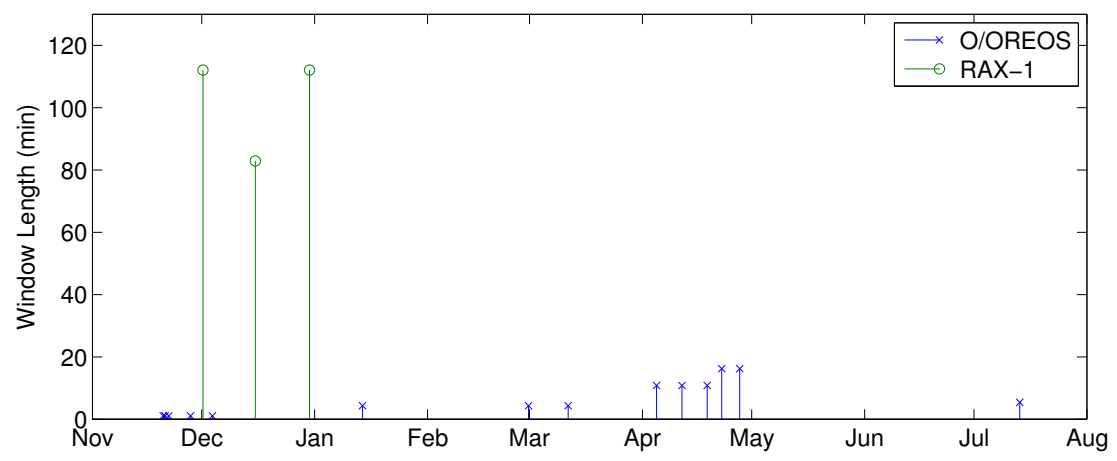

Figure 2: Availability of Flight Data from the O/OREOS and RAX-1 Nano Satellites. Several data sets over several months were available from O/OREOS and each set lasted a few minutes. Three data sets were available during the first month from RAX-1 and each set lasted over an hour. Data was recorded at $1 \mathrm{~Hz}$ during the collection windows.

two hours long. The distribution of available data from each spacecraft is shown in Figure 2, and sample data from the individual windows are illustrated in Figure 3 .

During the data windows, both O/OREOS and RAX-1 recorded the electrical currents flowing from their body mounted solar panels. Additionally, RAX-1 recorded data from its other onboard attitude sensors. The solar panel currents (for O/OREOS) and the photodiode readings (for RAX-1) were pre-processed to obtain estimates of the solar vector using the methods described in Ref. [10].

\section{The Gyro Free MEKF}

In this section a MEKF formulation is developed for application to O/OREOS. Specifically, the gyro-driven motion model in the original MEKF formulation is replaced with a model based on the spacecraft's attitude dynamics. Instead of estimating gyro bias, the MEKF developed in this system explicitly estimates the spacecraft angular velocity by modeling the spacecraft's angular acceleration.

The MEKF, first proposed in Ref. [11], was designed to overcome the two problems associated with filtering quaternions: maintaining unit length and avoiding singular state covariance matrices. The original MEKF will not be reproduced here although the author can find a full derivation in Ref. [4]. 

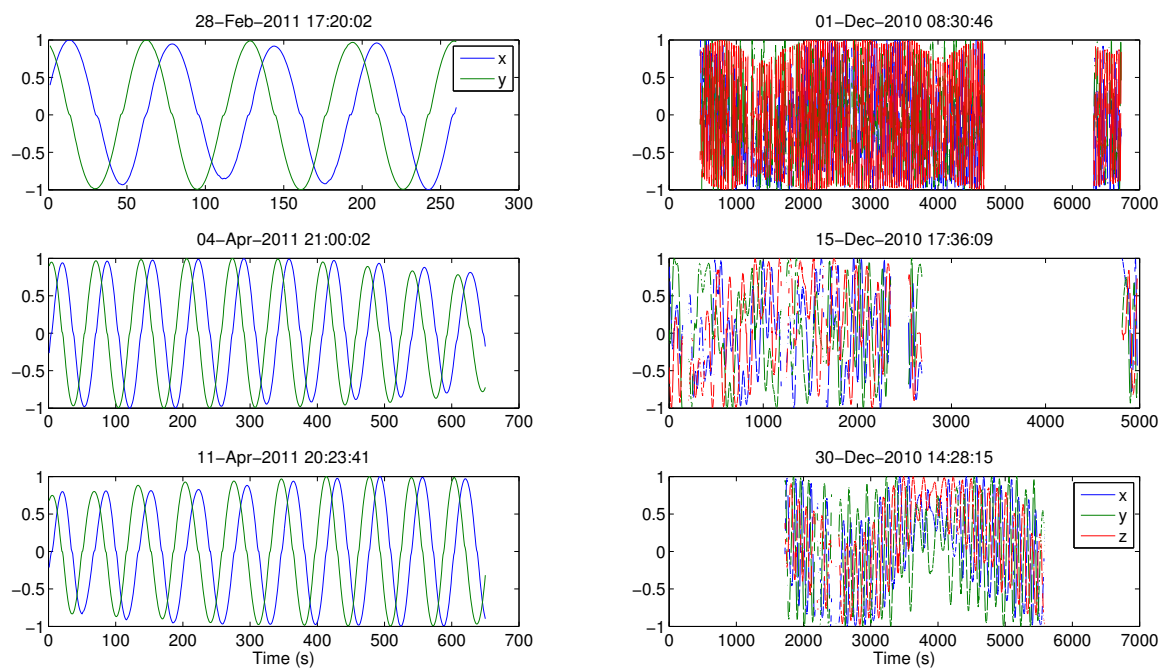

(a) O/OREOS

(b) RAX-1

Figure 3: Sample Solar Vector Data Sets from the O/OREOS and RAX-1 Nano Satellites. For O/OREOS, three example windows are plotted, each lasting a few minutes. For RAX-1, the three available windows are plotted, each lasting around two hours. The $x$ and $y$ components of the solar vector are plotted in blue and green respectively. For RAX-1, a $z$ component was also available and is plotted in red. On both spacecraft data was recorded at $1 \mathrm{~Hz}$.

\subsection{Quaternions}

In a MEKF, spacecraft attitude is represented using a unit quaternion as defined in Eqs. (1) and (2). The quaternion notation used in this work borrows heavily from that found in Ref. [3], where the scalar last convention is used.

$$
\begin{aligned}
q & \equiv\left[\begin{array}{c}
\varrho \\
q_{4}
\end{array}\right] \\
\varrho \in \mathbb{R}^{3}, q_{4} & \in \mathbb{R},\|q\|_{2}=1
\end{aligned}
$$

The direction cosine matrix, $A$, can be computed from a quaternion using Eq. (3),

$$
A(q)=\left(q_{4}^{2}-\|\varrho\|^{2}\right) \mathrm{I}_{3 \times 3}+\varrho \varrho^{\mathrm{T}}-2 q_{4}[\varrho \times]=\Xi^{\mathrm{T}}(q) \Psi(q)
$$


where the $\Xi$ and $\Psi$ matrices are defined in Eqs. (4) and (5),

$$
\begin{aligned}
& \Xi(q) \equiv\left[\begin{array}{c}
q_{4} \mathrm{I}_{3 \times 3}+[\varrho \times] \\
-\varrho^{\mathrm{T}}
\end{array}\right] \\
& \Psi(q) \equiv\left[\begin{array}{c}
q_{4} \mathrm{I}_{3 \times 3}-[\varrho \times] \\
-\varrho^{\mathrm{T}}
\end{array}\right]
\end{aligned}
$$

and the skew symmetric matrix, $[\times]$, is defined in Eq. (6).

$$
[\varrho \times] \equiv\left[\begin{array}{ccc}
0 & -\varrho_{3} & \varrho_{2} \\
\varrho_{3} & 0 & -\varrho_{1} \\
-\varrho_{2} & \varrho_{1} & 0
\end{array}\right]
$$

Finally, the non-commutative quaternion multiplication operator $\otimes$ is defined in Eqs. (7) and (8).

$$
\begin{aligned}
& q^{\prime} \otimes q=\left[\begin{array}{ll}
\Psi\left(q^{\prime}\right) & q^{\prime}
\end{array}\right] q=[\Xi(q) \quad q] q^{\prime} \\
& A\left(q^{\prime} \otimes q\right)=A\left(q^{\prime}\right) A(q)
\end{aligned}
$$

\subsection{Setup}

In a MEKF the spacecraft's true attitude, $q$, is composed of a deterministic reference quaternion and a stochastic error quaternion, $\delta q$, as given in Eq. (9).

$$
q=\delta q \otimes q_{\mathrm{ref}}
$$

The error quaternion, $\delta q$, is small and can be parameterized by a three component error vector, $a \in \mathbb{R}^{3}$. In a deviation from the derivation in Ref. [4], the approximation in Eq. (10) will be used in this work.

$$
\delta q=\delta q(a) \approx\left[\begin{array}{l}
a \\
1
\end{array}\right]
$$

The underlying spacecraft system and measurement equations used in a gyro free MEKF are defined in Eqs. (11) - 15.

$$
\begin{aligned}
\dot{q} & =\frac{1}{2} \omega \otimes q \\
\dot{q}_{\mathrm{ref}} & =\frac{1}{2} \omega_{\mathrm{ref}} \otimes q_{\mathrm{ref}} \\
\dot{a} & =\frac{1}{2}\left(\omega-\omega_{\mathrm{ref}}+a \times\left(\omega+\omega_{\mathrm{ref}}\right)\right) \\
\dot{\omega} & =f(q, \omega, \eta) \\
\tilde{c} & =A(q) c_{\mathrm{ECI}}+\nu
\end{aligned}
$$


The motion model is described by Eqs. (11)-(14). Eqs. (11) and (12) are the kinematic equations for the stochastic truth and deterministic reference states respectively, and Eq. (13) is the time derivative of the stochastic error vector. Eq. (14) describes the attitude dynamics, where $\eta$ are system noise terms with covariance $\Sigma_{\eta}$.

Eq. (15) is the measurement equation, where $c_{\mathrm{ECI}}$ denotes the unit vector pointing from the spacecraft to the sun in an inertial frame, $\tilde{c}$ represents the measurement of this vector in the body frame and $\nu$ represents measurement noise. Although both the measured and true solar vectors are unit vectors, assuming the measurement noise to be isotropic and additive, as in Eq (16), while technically imperfect, does not degrade filter performance[2].

$$
\begin{aligned}
\nu & \sim \mathcal{N}\left(0, \Sigma_{\nu_{c}}\right) \\
& \sim \mathcal{N}\left(0, \sigma_{c}^{2} \mathrm{I}_{3 \times 3}\right)
\end{aligned}
$$

\subsection{The Filter}

It is assumed that the spacecraft can measure the solar vector in the body frame, $\tilde{c}$, with measurement covariance $\Sigma_{\nu_{c}}$, and these measurements arrive at time $t_{k}$. The sun vector in the inertial frame, $c_{\mathrm{ECI}}$, which is a function of spacecraft orbital location and time of year, is assumed known.

In a gyro free MEKF, the estimated state is comprised of the error vector, $a$, and the spacecraft angular velocity, $\omega$. The error vector, $a$, is held at zero during the time update, requiring the expectation of the angular velocity to equal the reference angular velocity, $\hat{\omega}=\omega_{\text {ref. }}$ After a measurement is processed, the now non-zero error vector is incorporated into the attitude estimate using a multiplicative update.

For a spacecraft without gyros, Eqs. (17)-29) describe an MEKF. 
Time Update:

$$
\begin{aligned}
\dot{q}_{\mathrm{ref}}(t) & =\frac{1}{2} \hat{\omega}(t) \otimes q_{\mathrm{ref}}(t) \\
\hat{\omega}(t) & =f\left(q_{\mathrm{ref}}, \hat{\omega}, 0\right) \\
\dot{\hat{\Sigma}} & =F \hat{\Sigma}+\hat{\Sigma} F^{\mathrm{T}}+G Q G^{\mathrm{T}} \\
F(t) & =\left[\begin{array}{ll}
\left.\frac{\partial \dot{a}}{\partial a}\right|_{\hat{a}, \hat{\omega}} & \left.\frac{\partial \dot{a}}{\partial \omega}\right|_{\hat{a}, \hat{\omega}} \\
\left.\frac{\partial \dot{\omega}}{\partial a}\right|_{\hat{a}, \hat{\omega}} & \left.\frac{\partial \dot{\omega}}{\partial \omega}\right|_{\hat{a}, \hat{\omega}}
\end{array}\right]=\left[\begin{array}{cc}
-[\hat{\omega}(t) \times] & \frac{1}{2} \mathrm{I}_{3 \times 3} \\
\left.\frac{\partial f}{\partial a}\right|_{\hat{a}, \hat{\omega}} & \left.\frac{\partial f}{\partial \omega}\right|_{\hat{a}, \hat{\omega}}
\end{array}\right] \\
G(t) & =\left[\begin{array}{l}
\left.\frac{\partial \dot{a}}{\partial \eta}\right|_{\hat{a}, \hat{\omega}} \\
\left.\frac{\partial \dot{\omega}}{\partial \eta}\right|_{\hat{a}, \hat{\omega}}
\end{array}\right]=\left[\begin{array}{c}
0_{3 \times \ldots} \\
\left.\frac{\partial f}{\partial \eta}\right|_{\hat{a}, \hat{\omega}}
\end{array}\right] \\
Q & =\Sigma_{\eta}
\end{aligned}
$$

Measurement Update:

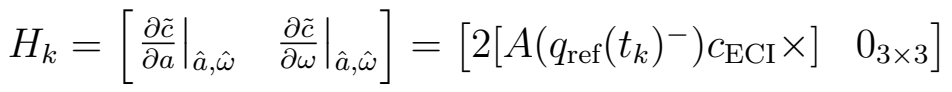

$$
\begin{aligned}
& K_{k}=\hat{\Sigma}\left(t_{k}\right)^{-} H_{k}^{\mathrm{T}}\left(H_{k} \hat{\Sigma}\left(t_{k}\right)^{-} H_{k}^{\mathrm{T}}+R\right)^{-1} \\
& R=\sigma_{c}^{2} \mathrm{I}_{3 \times 3} \\
& {\left[\begin{array}{c}
a_{k} \\
\Delta \omega_{k}
\end{array}\right]=K_{k}\left(\tilde{c}_{k}-A\left(q_{\mathrm{ref}}\left(t_{k}\right)^{-}\right) c_{\mathrm{ECI}}\right)} \\
& q_{\mathrm{ref}}\left(t_{k}\right)^{+}=\delta q\left(a_{k}\right) \otimes q_{\mathrm{ref}}\left(t_{k}\right)^{-} \\
& \hat{\omega}\left(t_{k}\right)^{+}=\Delta \omega_{k}+\hat{\omega}\left(t_{k}\right)^{-} \\
& \hat{\Sigma}\left(t_{k}\right)^{+}=\left(\mathrm{I}_{6 \times 6}-K_{k} H_{k}\right) \hat{\Sigma}\left(t_{k}\right)^{-}
\end{aligned}
$$

In Eqs. (17)-(29) an estimate of a variable is denoted with a circumflex $\left(^{\wedge}\right)$ and a measurement of a variable is denoted with a tilde $\left(^{\sim}\right)$. Measurements arrive at time $t_{k}$. A superscript minus $\left(^{-}\right)$denotes an estimate of a variable prior to a measurement being incorporated, and a superscript plus $\left(^{+}\right)$denotes an estimate after a measurement has been incorporated.

\subsection{Comparison to Original MEKF Formulation}

In the original MEKF derivation, the angular velocity is read directly from onboard gyros and uncertainties arise from the gyro noise plus the gyro bias

state covariance. In the gyro free MEKF the angular velocity is a state of the 
filter. Angular velocity uncertainty is quantified by the state covariance and is path dependent, driven by uncertainties in the dynamics model, uncertainties in the measurements, and the time history of measurements.

As the underlying dynamics are nonlinear and may be time variant, determining whether a given dynamics model would provide a more or less accurate estimate of angular velocity than a given gyro is not possible in closed form. For a specific path, Cramér-Rao lower bounds 12] could be computed for both cases but as these are single-sided bounds they can only provide an indication of relative accuracy.

\subsection{Attitude Dynamics}

So far in this section the problem of actually acquiring an attitude dynamics model is yet to be addressed. Obtaining an accurate rotational dynamics model for even a simple spacecraft with rigid body dynamics is not a straightforward problem[3], and this difficulty has been a driver of the historic reliance on using gyros to drive the motion model.

Recently a new method has been demonstrated[5] that can calibrate the parameters of a spacecraft attitude dynamics model. This method determined a set of initial conditions and model parameters that would best fit observed data by forming and solving a non-convex optimization problem. Developing the parametric rotational dynamics model used in the optimization required a detailed analysis of the external torques acting on the orbiting spacecraft [10]. This approach is used with the attitude dynamics formulated in the Section 4

\subsection{Reincorporating Gyros}

Although the filter developed in this section was originally designed for application where no gyros are available, if gyros were available the data from these can easily be incorporated into the new filter with only minor modifications. Such a design would be useful when the noise in the gyros is similar to the uncertainty in the attitude dynamics model. In this situation, using a combination of the two angular velocity estimation sources should result in a better estimate than using just one. Conversely, in situations where one source has much less noise or uncertainty than the other, using both will not significantly improve the accuracy of estimate over just using the less uncertain one.

To include data from gyros while still using an attitude dynamics based motion model, the gyro readings should be incorporated in the measurement 
update, rather than as part of the motion update. The gyro bias still needs to be estimated and is augmented to the state.

\section{Gyro-Free MEKF for a Passively Magnetically Stabilized Nano Satellite}

In this section the gyro-free MEKF described previously is implemented for the passively magnetically stabilized nano satellites studied in this paper. A spacecraft dynamics model is derived for the satellites and this model is then substituted into the filter equations.

\subsection{Spacecraft Dynamics}

Eq. (30) is the equation of motion, derived from Euler's Equation, for a passively magnetically stabilized spacecraft.

$$
0=I \dot{\omega}+\omega \times I \omega+\mu_{0}(H \times M)-T_{\text {dist }}
$$

In Eq. (30), $\mu_{0}$ is the permeability of free space which equals $4 \pi \times 10^{-7} \mathrm{H} \mathrm{m}^{-1}$ in the SI unit system, $\omega$ is the angular velocity, $I$ is the moment of inertia tensor, $H$ is the external magnetic field, $M$ is the total dipole, and $T_{\text {dist }}$ is the external disturbance torques, all expressed in a body fixed frame. The torques acting on the spacecraft have deliberately been split between those arising from the passive magnetic stabilization system and those arising from other sources. As can be seen from Table 1, which lists the major torques acting on the nano satellites in this study, these disturbance torques are several orders of magnitude less than those arising from the magnetic system, and will not be modeled.

\begin{tabular}{lc}
\hline Torque Source & Range of Values $(\mathrm{Nm})$ \\
\hline Magnetic Dipole & $2 \times 10^{-5}$ to $5 \times 10^{-4}$ \\
Gravity Gradient & $2 \times 10^{-8}$ to $3 \times 10^{-8}$ \\
Aerodynamic Drag & $1 \times 10^{-9}$ to $8 \times 10^{-7}$ \\
Solar Radiation Pressure & $2 \times 10^{-9}$ to $2 \times 10^{-8}$ \\
\hline
\end{tabular}

Table 1: Torques Acting on LEO Nano Satellite. For the computations in this table, a $3 \mathrm{U}$ CubeSat with no deployable structures in a $400 \mathrm{~km}-650 \mathrm{~km}$ altitude low earth orbit (LEO) was assumed. 
The external magnetic field in the body frame, $H$, is computed from the assumed known value in an inertial frame using Eq. (31), where $q$ is the unit quaternion describing the spacecraft's attitude.

$$
H=A(q) H_{\mathrm{ECI}}
$$

The external magnetic field in an inertial frame, $H_{\mathrm{ECI}}$, can be computed using the IGRF[13] model.

A passive magnetic stabilization system consists of permanent dipoles that provide alignment to the Earth's magnetic field, and permeable rods that provide damping during the post-separation de-tumble phase. The total dipole, $M$, can be computed using Eq. (32).

$$
M=M_{\mathrm{P}}+\sum_{i=1}^{m} n_{i} \frac{B_{i}(t) V_{i}}{\mu_{0}}
$$

In Eq. (32) $M_{\mathrm{P}}$ is the permanent dipole, and $B_{i}$ and $V_{i}$ are the induced flux density in and the volume of the $i$ th of $m$ permeable rods respectively. The unit vector $n_{i}$ is parallel to the long axis of the rod.

An attitude determination capability is most important during the science phase of the mission, when the spacecraft has finished de-tumbling and the permeable rods are providing minimal damping. During this phase the contribution from the permanent dipole dominates the total dipole, and so it will be assumed that $M \approx M_{\mathrm{P}}$. The magnetic torques arising from the unmodeled permeable rod dipoles are larger than the other disturbance torques acting on the spacecraft, but are still at least an order of magnitude less than that arising from the permanent dipole.

The parameters of passive magnetic stabilization systems have been shown to be hard to characterize prior to launch [14, 15], making the utilization of on orbit calibration techniques [5] essential for good filter performance.

Determining whether the calibration results in a dynamics model that is sufficiently accurate to achieve desired filter performance is a non-trivial problem that cannot be answered in closed form. In Section 5 of this paper the impact of model accuracy on filter performance is explored empirically by varying the relative magnitude of un-modeled torques.

\subsection{Estimating the Solar Vector}

In this paper it is assumed that the only measurement available is an estimate of the solar vector. This can be obtained from differential solar 
270 illustrates the general process, whereby raw readings are taken, scaled based on recent maxima to account for degradation, and then opposing panels inverted, before finally scaling to a unit-length vector.
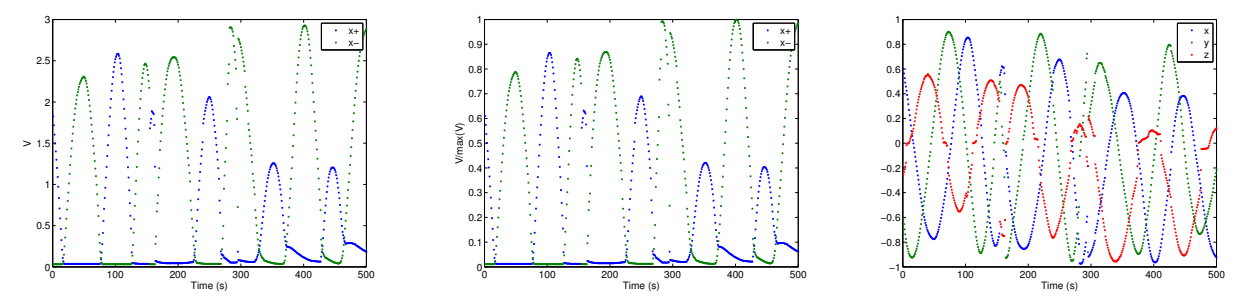

(a) Raw Sensor Readings (b) Scaled Sensor Read- (c) Estimated Sun Vector (taken from $+x$ and $-x$ ings (taken from $+x$ and panels)

Figure 4: Example of estimating sun vector from raw sensor readings

As can be seen from Figure 4(c) the final vector estimate is not a clean estimate. Neither solar panel currents nor photodiode voltages are pure cosine estimators. Further readings taken with shallow glancing angles (close to $90^{\circ}$ to the surface normal) are especially noisy, leading to data corruption close to zero-crossings. Despite all these problems, as will be seen in the results presented in Section 6, this estimate is still sufficient for the filter to provide good attitude estimates.

The estimate of the solar vector in the body frame can be related to that in the inertial frame using Equation (33), where $q$ is the unit quaternion describing the spacecraft's attitude.

$$
c=q \otimes c_{\mathrm{ECI}} \otimes q^{-1}=A(q) c_{\mathrm{ECI}}
$$

The solar vector in an inertial frame is a function of the sun's position in 285 inertial space with respect to the spacecraft, as in Equation (34), and varies predominantly with the time of year.

$$
c_{\mathrm{ECI}}=\left(\frac{r_{\mathrm{sun}}-r_{\mathrm{s} / \mathrm{c}}}{\left\|r_{\mathrm{sun}}-r_{\mathrm{s} / \mathrm{c}}\right\|}\right)_{\mathrm{ECI}}
$$

\subsection{Setup}

The system and measurement equations for a gyro-free MEKF for a passively magnetically stabilized spacecraft using solar vector measurements are 


\begin{tabular}{cll}
\hline Term & Description & Value \\
\hline$\sigma_{T}$ & torque disturbances & $7.5 \times 10^{-6} \mathrm{~N} \mathrm{~m}^{-1}$ \\
$\sigma_{H}$ & errors in magnetic field & $2.4 \times 10^{-2} \mathrm{~A} \mathrm{~m}^{-1}$ \\
\hline
\end{tabular}

Table 2: Assumed Noise in System Dynamics Model. The torque noise is based on the magnitude of the largest un-modeled torques, which arise from the magnetically permeable material. The error in the magnetic field is based on recommendations from the publishers of the IGRF model.

given in Eqs. 35- 39 .

$$
\begin{aligned}
\dot{q} & =\frac{1}{2} \omega \otimes q \\
\dot{q}_{\mathrm{ref}} & =\frac{1}{2} \hat{\omega} \otimes q_{\mathrm{ref}} \\
\dot{a} & =\frac{1}{2}(\omega-\hat{\omega}+a \times(\omega+\hat{\omega})) \\
\dot{\omega} & =I^{-1}\left(\mu_{0}\left(M_{\mathrm{P}} \times\left(A(q) H_{\mathrm{ECI}}+\eta_{2}\right)\right)-\omega \times I \omega+\eta_{1}\right) \\
\tilde{c} & =A(q) c_{\mathrm{ECI}}+\nu
\end{aligned}
$$

Eqs. (35)-(37) are the same kinematic equations presented previously. Eq. (38), the attitude dynamics model, was derived by combining and rearranging Eqs. (30) and (31) and adding noise terms. The noise term $\eta_{1}$ models unknown disturbance torques, and the noise term $\eta_{2}$ models errors in the IGRF field, including those arising from orbital position errors. Both are assumed isotropic, with standard deviations $\sigma_{T}$ and $\sigma_{H}$ respectively, as given in Eqs. (40) and (41), and suitable values for $\sigma_{T}$ and $\sigma_{H}$ are given in Table 2 ,

$$
\begin{array}{ll}
\mathrm{E}\left[\eta_{1}\right]=0, & \mathrm{E}\left[\eta_{1} \eta_{1}^{\mathrm{T}}\right]=\sigma_{T}^{2} \mathrm{I}_{3 \times 3} \\
\mathrm{E}\left[\eta_{2}\right]=0, & \mathrm{E}\left[\eta_{2} \eta_{2}^{\mathrm{T}}\right]=\sigma_{H}^{2} \mathrm{I}_{3 \times 3}
\end{array}
$$

Eq. (39) is the same measurement equation as used throughout this paper.

For the passively magnetically stabilized nano satellites studied in this paper, Eqs. (42)-54) describe a gyro free MEKF. 
Time Update:

$$
\begin{aligned}
\dot{q}_{\mathrm{ref}}(t) & =\frac{1}{2} \hat{\omega}(t) \otimes q_{\mathrm{ref}}(t) \\
\dot{\hat{\omega}} & =I^{-1}\left(\mu_{0}\left(M_{\mathrm{P}} \times\left(A\left(q_{\mathrm{ref}}\right) H_{\mathrm{ECI}}\right)\right)-\hat{\omega} \times I \hat{\omega}\right) \\
\dot{\hat{\Sigma}} & =F \hat{\Sigma}+\hat{\Sigma} F^{\mathrm{T}}+G Q G^{\mathrm{T}} \\
F(t) & =\left[\begin{array}{ll}
\left.\frac{\partial \dot{a}}{\partial a}\right|_{\hat{a}, \hat{\omega}} & \left.\frac{\partial \dot{a}}{\partial \omega}\right|_{\hat{a}, \hat{\omega}} \\
\left.\frac{\partial \dot{\omega}}{\partial a}\right|_{\hat{a}, \hat{\omega}} & \left.\frac{\partial \dot{\omega}}{\partial \omega}\right|_{\hat{a}, \hat{\omega}}
\end{array}\right] \\
& =\left[\begin{array}{ll}
2 \mu_{0} I^{-1}\left[M_{\mathrm{P}} \times\right] \\
-\left[A\left(q_{\mathrm{ref}}\right) H_{\mathrm{ECI}} \times\right] & I^{-1}(-[\hat{\omega} \times] I+[I \hat{\omega} \times])
\end{array}\right] \\
G & =\left[\begin{array}{ll}
\left.\frac{\partial \dot{a}}{\partial \eta_{1}}\right|_{\hat{a}, \hat{\omega}} & \left.\frac{\partial \dot{a}}{\partial \eta_{2}}\right|_{\hat{a}, \hat{\omega}} \\
\left.\frac{\partial \dot{\omega}}{\partial \eta_{1}}\right|_{\hat{a}, \hat{\omega}} & \left.\frac{\partial \dot{\omega}}{\partial \eta_{2}}\right|_{\hat{a}, \hat{\omega}}
\end{array}\right]=\left[\begin{array}{cc}
0_{3 \times 3} & 0_{3 \times 3} \\
I^{-1} & \mu_{0} I^{-1}\left[M_{\mathrm{P}} \times\right]
\end{array}\right] \\
Q & =\left[\begin{array}{cc}
\sigma_{T}^{2} \mathrm{I}_{3 \times 3} & 0_{3 \times 3} \\
0_{3 \times 3} & \sigma_{H}^{2} \mathrm{I}_{3 \times 3}
\end{array}\right]
\end{aligned}
$$

Measurement Update:

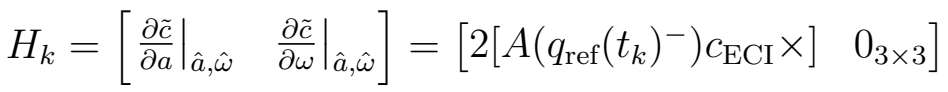

$$
\begin{aligned}
& K_{k}=\hat{\Sigma}\left(t_{k}\right)^{-} H_{k}^{\mathrm{T}}\left(H_{k} \hat{\Sigma}\left(t_{k}\right)^{-} H_{k}^{\mathrm{T}}+R\right)^{-1} \\
& R=\sigma_{c}^{2} \mathrm{I}_{3 \times 3} \\
& {\left[\begin{array}{c}
a_{k} \\
\Delta \omega_{k}
\end{array}\right]=K_{k}\left(\tilde{c}_{k}-A\left(q_{\mathrm{ref}}\left(t_{k}\right)^{-}\right) c_{\mathrm{ECI}}\right)} \\
& q_{\mathrm{ref}}\left(t_{k}\right)^{+}=\delta q\left(a_{k}\right) \otimes q_{\mathrm{ref}}\left(t_{k}\right)^{-} \\
& \hat{\omega}\left(t_{k}\right)^{+}=\Delta \omega_{k}+\hat{\omega}\left(t_{k}\right)^{-} \\
& \hat{\Sigma}\left(t_{k}\right)^{+}=\left(\mathrm{I}_{6 \times 6}-K_{k} H_{k}\right) \hat{\Sigma}\left(t_{k}\right)^{-}
\end{aligned}
$$

\subsection{Implementation Notes}

Eqs. (42) and (43) can be integrated using a Runge-Kutta 4th order algorithm, with time step chosen such that $\|\omega d t\|_{2} \ll 1$. Although Eq. (44) can be integrated as written, maintaining $\hat{\Sigma}$ to be positive definite requires ad hoc matrix re-projections. Instead, the estimate of the covariance matrix can be updated using a state transition matrix as in Eq. 55, which maintains 
$\hat{\Sigma}>0$.

$$
\hat{\Sigma}\left(t_{1}\right)=\Phi_{t_{0}, t_{1}} \hat{\Sigma}\left(t_{0}\right) \Phi_{t_{0}, t_{1}}^{\mathrm{T}}+\int_{t_{0}}^{t_{1}} G(\tau) Q(\tau) G^{\mathrm{T}}(\tau) d \tau
$$

The state transition matrix $\Phi$ is computed using Eq. (56).

$$
\Phi_{t_{0}, t_{1}}=\exp \left(F\left(t_{0}\right)\left(t_{1}-t_{0}\right)\right)
$$
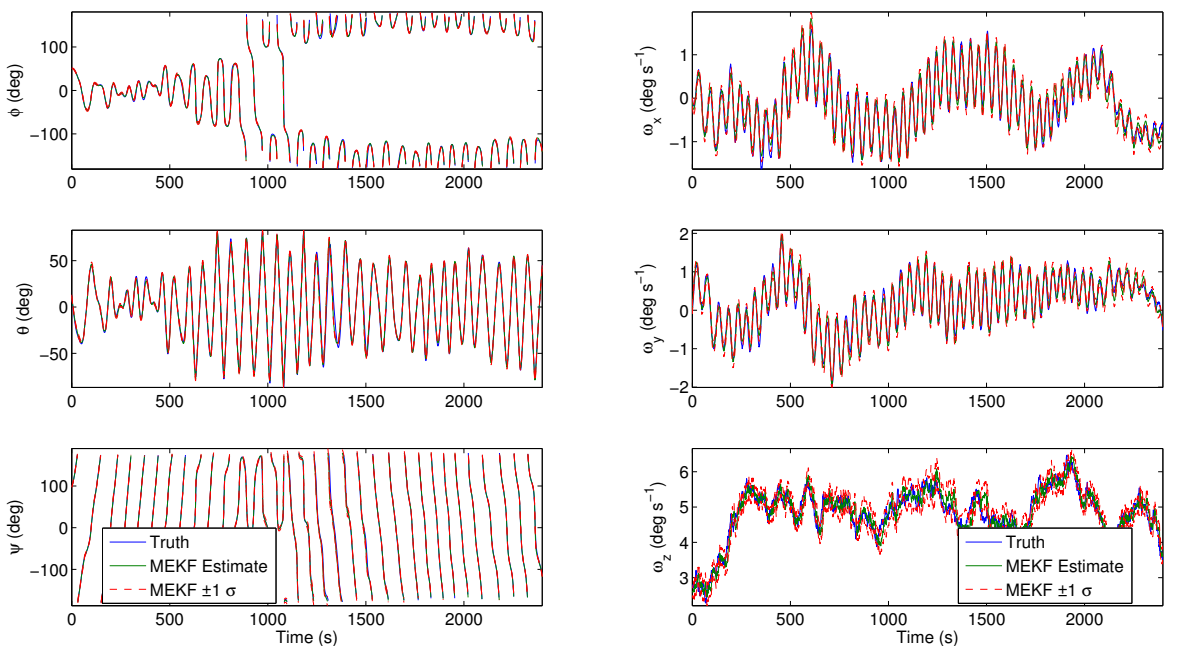

(a) Euler Angles, $(\phi, \theta, \psi)$

(b) angular velocity, $\omega$

Figure 5: Performance of the MEKF Attitude Filter in Simulation. Plotted in blue is the underlying simulated truth data. Plotted in green is the estimate as generated by the MEKF. Plotted in dashed red are the $\pm 1 \sigma$ bounds as determined by the MEKF. The two estimates lie close to exactly on top of each other.

\section{Results in Simulation}

In this section the filter derived in Section 4 is tested in simulation, before application to real flight data in Section 6. The simulation mirrors the conditions experienced by the satellites studied: a $650 \mathrm{~km}$ altitude low earth orbit at $72^{\circ}$ inclination. 

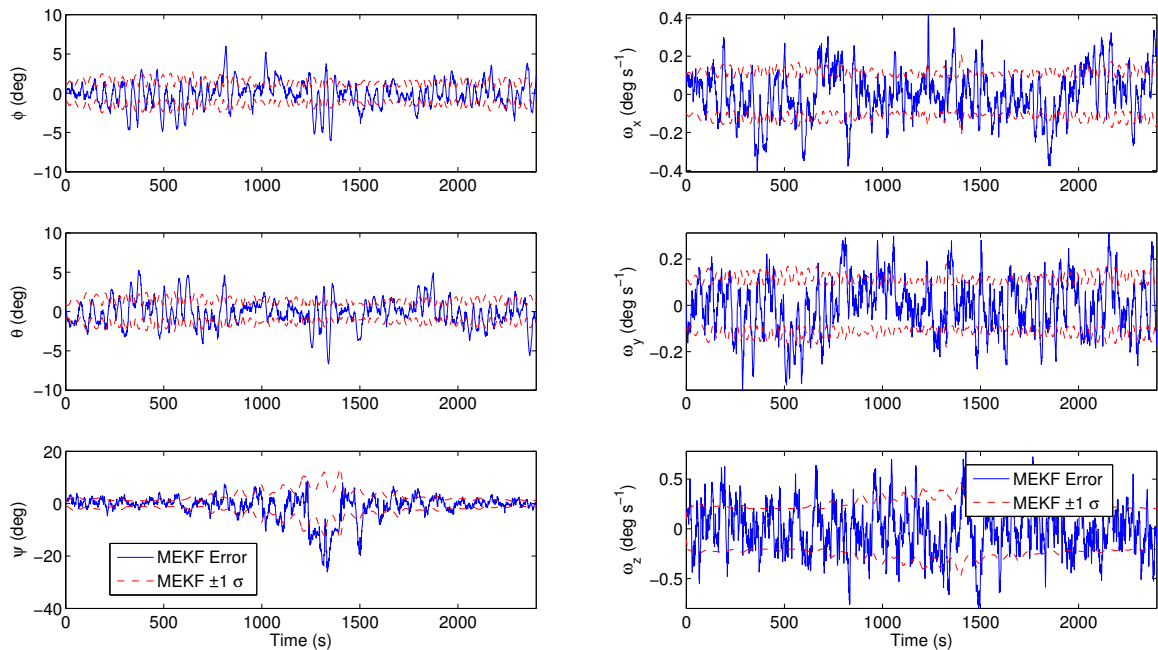

(a) Euler Angles, $(\phi, \theta, \psi)$

(b) angular velocity, $\omega$

Figure 6: Errors of the MEKF Attitude Filter in Simulation. Plotted in blue is the error of the MEKF, defined as the difference between the simulated truth data and the MEKF estimate. Plotted in dashed red are the $\pm 1 \sigma$ bounds as determined by the MEKF. The errors in each Euler angle are nominally $3^{\circ}$ to $4^{\circ}$, but with a divergence around $t=1300 \mathrm{~s}$, when errors increase to nearer $5^{\circ}$ in $\phi$ and $\theta$ and over $20^{\circ}$ in $\psi$. This divergence is explained by looking at the observability of the problem, as described in Section 5 . Errors in angular velocities are typically $0.1^{\circ} \mathrm{s}^{-1}$ in $x$ and $y$ components and $0.3^{\circ} \mathrm{s}^{-1}$ in the $z$ component. In both attitude and attitude error, the predicted $\pm 1 \sigma$ bound matches the actual errors well, an attractive feature of a filter.

\subsection{Nominal Results}

The filter's performance in simulation is illustrated in Figures 5 and 6 Artificial disturbances and measurement noise were added, based on typical values for the low earth orbiting $3 \mathrm{U}$ CubeSats studied in this paper, and specific values are detailed in Table 3. No additional tuning of the process noise in the MEKF was required to achieve convergence.

Looking at Figure 6(a), which plots the filter error, typical attitude errors on each axis are of the order of a few degrees. Looking at Figure 6(b), typical attitude rate errors are of the order of $0.1^{\circ} \mathrm{s}^{-1}$ around the $x$ and $y$ axes and $0.2^{\circ} \mathrm{s}^{-1}$ around the $z$ axis.

Figure 7 plots the total attitude error, or pointing knowledge, of the MEKF with simulated data. The estimated $1 \sigma$ pointing error shown in Fig- 


\begin{tabular}{cll}
\hline Term & Description & Value \\
\hline$\sigma_{T}$ & torque disturbances & $7.5 \times 10^{-6} \mathrm{~N} \mathrm{~m}^{-1}$ \\
$\sigma_{H}$ & errors in magnetic field & $2.4 \times 10^{-2} \mathrm{~A} \mathrm{~m}^{-1}$ \\
$\sigma_{c}$ & solar vector measurement noise & $4.0 \times 10^{-2}$ \\
\hline
\end{tabular}

Table 3: Noise and Uncertainty Values used in MEKF for Testing Filter Performance. The torque noise is based on the magnitude of the largest un-modeled disturbance torques. The error in the magnetic field is based on recommendations from the publishers of the IGRF model. The solar vector measurement noise was described in Section 4 .

ure 7 is a conservative bound that assumes the largest error of the individual axis errors is distributed isotropically across all axes. As overall pointing error is a 2-norm measurement, the $\chi^{2}$ distribution function with three degrees of freedom is used to find the location of the $31.7 \%$ tail (two-sided $1 \sigma$ standard normal), which occurs at $\sqrt{3.5627} \sigma_{\max }$.

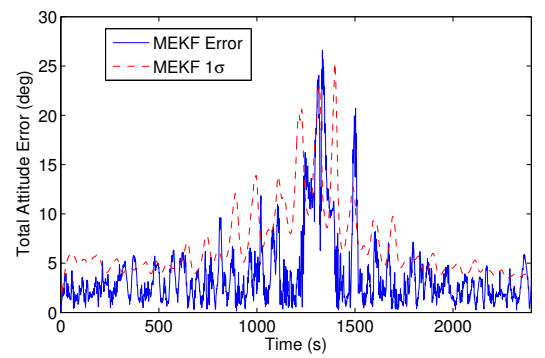

Figure 7: MEKF Total Attitude Error in Simulation. Plotted in blue is the total attitude error of the MEKF, defined as the angular difference between the simulated truth attitude and the MEKF estimated attitude. Plotted in dashed red is the $1 \sigma$ bound of this estimate. Total attitude error is typically $5^{\circ}$, but grows to in excess of $20^{\circ}$ around $t=1200 \mathrm{~s}$. This growth in attitude error is explained by examining the observability of the problem, as described in Section 5. Throughout the predicted $1 \sigma$ bound matches the actual errors well.

\subsection{Observability}

In the simulation results presented above, the filter typically exhibits total attitude error of less than $5^{\circ}$. However at around $t=1200 \mathrm{~s}$, performance degrades significantly and attitude error deteriorates to $20^{\circ}$. This poor filter performance can be explained by examining the orientation of the spacecraft spin axis and sun vector at that time. Figure 8(b) plots the angle between the spin vector and the solar vector. When these two vectors are aligned, the 
spin rate cannot be observed and spacecraft attitude becomes unobservable. When the two vectors are close to aligned, observability decreases leading to the poor filter performance. Once the geometry improves the filter is able to return to improved performance. Importantly during this period the filter's error estimate also deteriorates, so the filter does not become overly confident in the attitude estimate it produces.

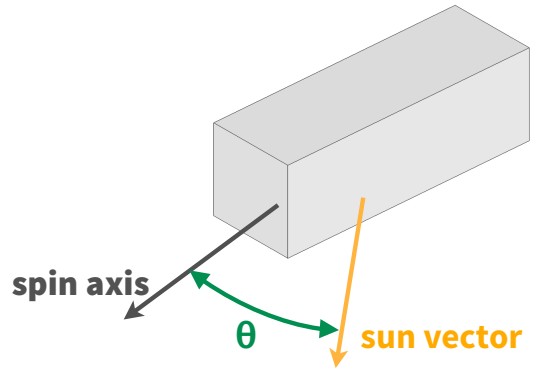

(a) Diagram

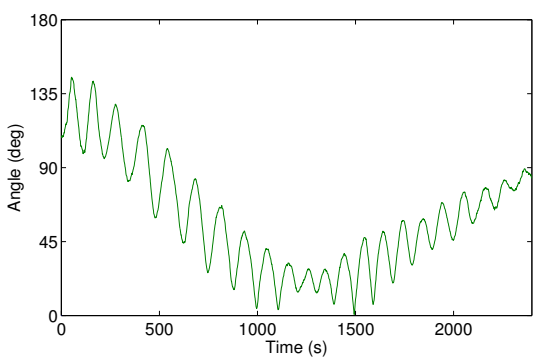

(b) Angle between spin vector and sun vector

Figure 8: Spacecraft Observation Geometry. The angle between the spin axis and the sun vector is illustrated in Figure 8(a). The variation of this angle during the simulation is plotted in Figure 8(b). When this angle is close to $0^{\circ}$ or $180^{\circ}$, the observability is expected to deteriorate. At around $t=1200 \mathrm{~s}$ the spin vector and the sun vector become close to aligned, and filter performance deteriorates.

The simulated spacecraft was assumed to have a permanent dipole aligned with the $z$ axis of the spacecraft, so the nominal spin axis is expected to also be the $z$ axis, and this is confirmed by Figure 5(b), where $\omega_{z}$ is typically $5^{\circ} \mathrm{s}^{-1}$, whereas $\omega_{x}$ and $\omega_{y}$ are nominally $0^{\circ} \mathrm{s}^{-1}$. If the deterioration in filter performance is indeed an observability issue, it would be expected that the largest angular errors would be around the $z$ axis, or Euler angle $\psi$. This is confirmed by examining Figure 6(a). At around $t=1200 \mathrm{~s}$, the error in $\psi$ diverges to over $20^{\circ}$, whereas the deviations in $\phi$ and $\theta$ are not noticeably larger at this point. The total attitude error in Figure 7 at $t=1200 \mathrm{~s}$ mostly comes from the error in $\psi$, confirming the observability issue around that axis.

\subsection{Model Accuracy}

As mentioned previously in Section 3, filter performance is dependent on the accuracy of the dynamics model. To demonstrate the impact of model 


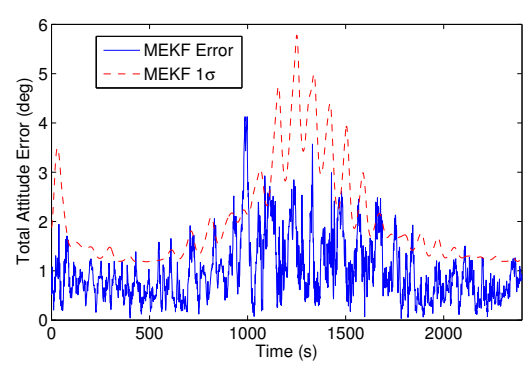

(a) $10 \times$ reduction

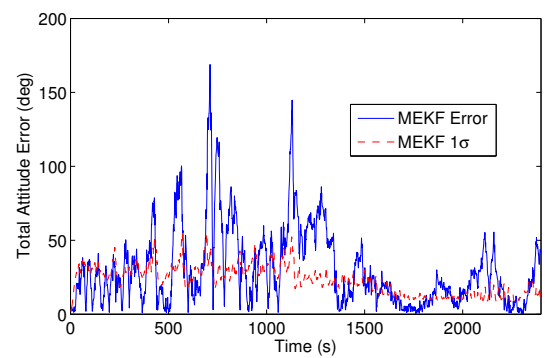

(c) $10 \times$ increase

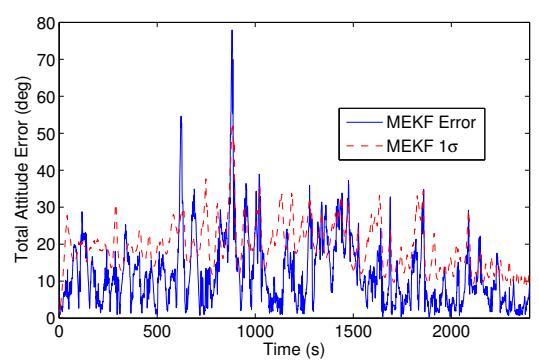

(b) $5 \times$ increase

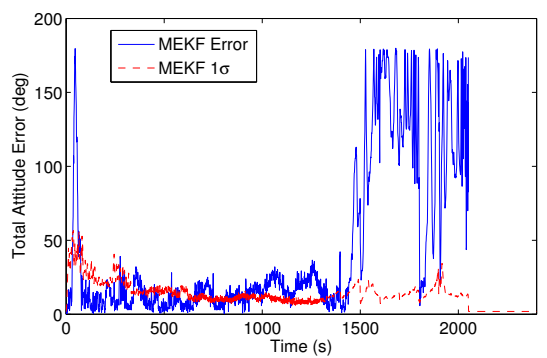

(d) $50 \times$ increase

Figure 9: Impact of Model Accuracy on Filter Error. The magnitude of the disturbance torques, $\sigma_{T}$, was varied in simulation. Plotted in blue is the error of the MEKF, defined as the difference between the simulated truth data and the MEKF estimate. Plotted in dashed red are the $\pm 1 \sigma$ bounds as determined by the MEKF. The accuracy of the filter is dependent on the accuracy of the system model. With a ten fold decrease in the magnitude of model disturbances, pointing accuracy is improved to $1^{\circ}$ typical. With a five times increase in model disturbances, typical pointing errors increase to $10^{\circ}$ typical. In both cases estimates of filter accuracy are internally consistent. At a ten times increase, errors regularly exceed $50^{\circ}$ and at fifty times the estimate no longer reliably converges. In both latter cases filter accuracy is overestimated internally.

accuracy, the filter was run with several different simulated model accuracies. For the passively magnetically stabilized spacecraft studied in this paper the largest uncertainty arises from the unknown disturbance torques. Model accuracy can be effectively varied by changing the assumed magnitude of these torque disturbances, $\sigma_{T}$, and the results are illustrated in Figure 9. For consistency, the process noise in the filter is also varied as the purpose is to test known model accuracy.

350 For small deviations in the magnitude of disturbance torques, overall filter error increases or decreases as expected. An order of magnitude reduction 
in the size of disturbance torques reduces typical errors to only $1^{\circ}$. An order of magnitude increase in the size of un-modeled disturbance torques increases filter errors to over $50^{\circ}$, and the filter's internal accuracy estimate is no longer consistent with the actual accuracy. Further, if the quality of the model is deteriorated even further, the filter is no longer able to converge and no estimate is obtained.

\section{Results}

In this section the filter derived in Section 4 is applied to actual flight data from NASA Ames Research Center's O/OREOS and the University of Michigan's RAX-1. The attitude estimates for RAX-1 were verified by comparing to estimates generated using traditional methods that incorporated all of that satellite's onboard attitude sensors.

For both spacecraft, values for the inertia $I$ and permanent dipole $M_{\mathrm{P}}$ were estimated using the batch calibration method referenced previously [5]. These calibrated values are reproduced in Table 4. Initial filter uncertainties

\begin{tabular}{cccc}
\hline Spacecraft & Inertia Tensor, $I\left(\mathrm{~kg} \mathrm{~m}^{2}\right)$ & Permanent Dipole, $M_{P}(\mathrm{Am})$ \\
\hline O/OREOS & {$\left[\begin{array}{ccc}0.05 & 0 & 0 \\
0 & 0.05 & 0 \\
0 & 0 & 0.01\end{array}\right]$} & {$\left[\begin{array}{l}0.30 \\
0.00 \\
17.4\end{array}\right]$} \\
\hline RAX-1 & {$\left[\begin{array}{lll}0.0185 & 0.0000 & 0.0001 \\
0.0000 & 0.0183 & 0.0004 \\
0.0001 & 0.0004 & 0.0043\end{array}\right]$} & {$\left[\begin{array}{l}0.14 \\
0.02 \\
1.09\end{array}\right]$} \\
\hline
\end{tabular}

Table 4: Spacecraft Physical Properties. The inertia tensor, $I$, and permanent dipole $M_{P}$ for the two spacecraft studied in this paper, determined using the calibration techniques described in Ref. [5]

were set to $\sigma_{a_{0}}=8.7 \times 10^{-3} \mathrm{rad}$ and $\sigma_{\omega_{0}}=1.7 \times 10^{-3} \mathrm{rad} \mathrm{s}^{-1}$ which correspond to $1^{\circ}$ in angle and $0.1^{\circ} / \mathrm{s}$ in rate, respectively.

\subsection{O/OREOS}

Figure 10 shows the filter estimates of attitude and attitude rate, using only the solar vector data from the O/OREOS nano satellite. The flight 
data was recorded in late April 2011 and O/OREOS was nominally spinning around its long $(z)$ axis.
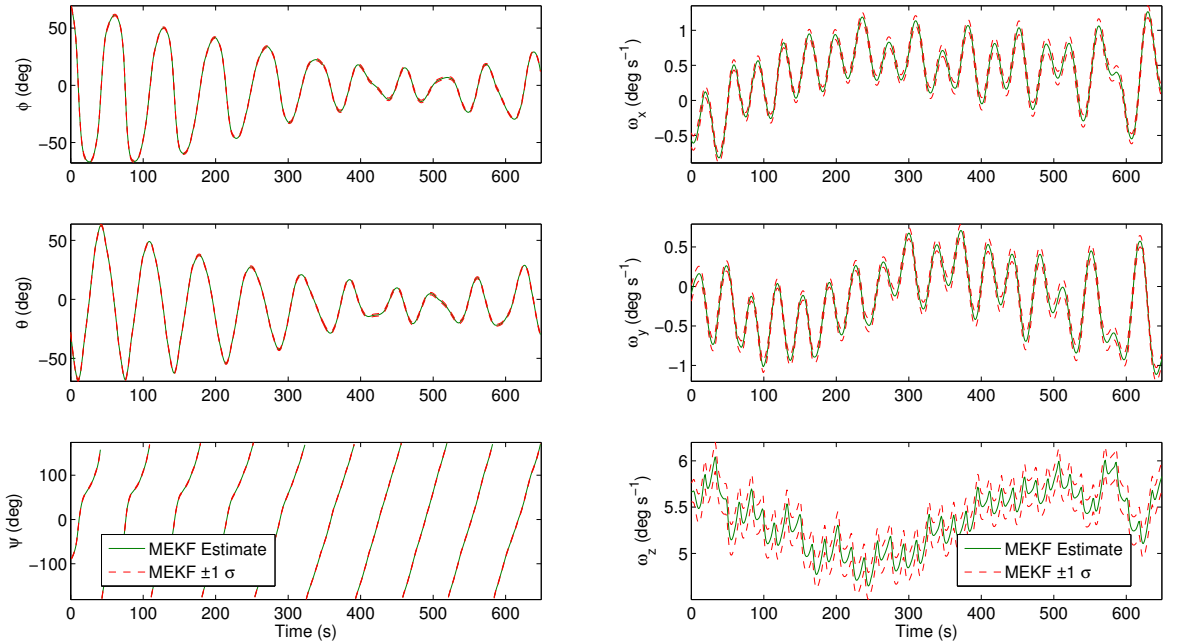

(a) Euler Angles, $(\phi, \theta, \psi)$

(b) angular velocity, $\omega$

Figure 10: Performance of the MEKF Attitude Filter for O/OREOS. Plotted in green is the estimate as generated by the MEKF. Plotted in dashed red is the $\pm 1 \sigma$ bound as determined by the filter. No truth data is available for O/OREOS and these attitude profiles cannot be verified.

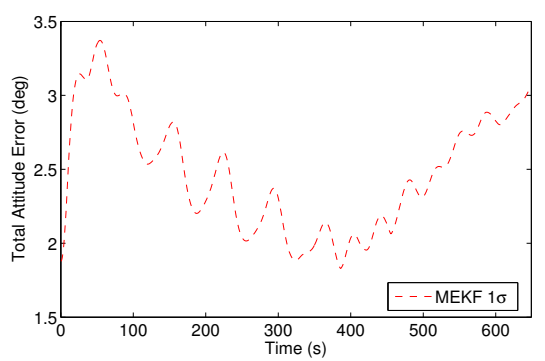

Figure 11: MEKF Total Attitude Error for O/OREOS. Plotted in dashed red is the $1 \sigma$ total attitude error. The filter claims performance of around $3^{\circ}$; however as no truth data is available for O/OREOS this performance cannot be verified.

As the only flight data available from O/OREOS are the solar panel currents, no verification of the attitude estimates are available. Attitude de- 
termination accuracy was estimated by the filter at around $3^{\circ}$, as illustrated in Figure 11 .
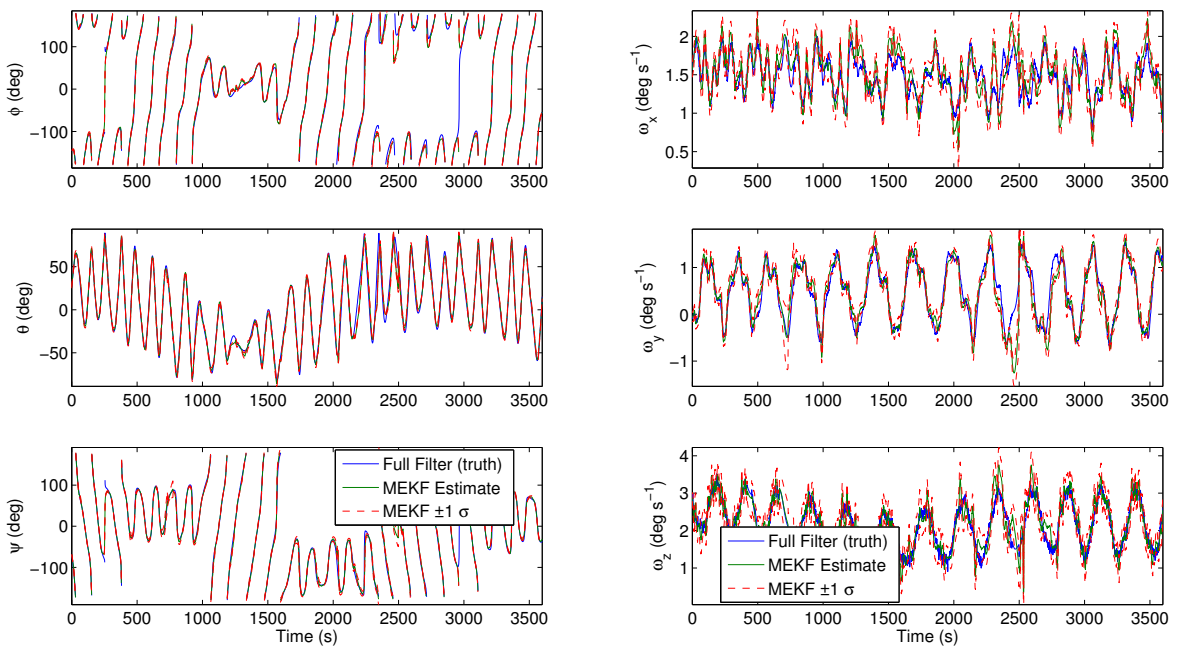

(a) Euler Angles, $(\phi, \theta, \psi)$

(b) angular velocity, $\omega$

Figure 12: Performance of the MEKF Attitude Filter for RAX-1. Plotted in blue is the full filter, a truth metric. Plotted in green is the estimate of the MEKF filter and in dashed red the $\pm 1 \sigma$ bound as determined by the filter. The MEKF estimate agrees with the full filter well, except for some divergence around $t=2000 \mathrm{~s}$. The full filter is a recursive attitude filter that uses all available bus measurements to achieve a best estimate of attitude.

\section{2. $R A X-1$}

Figure 12 shows the filter estimates of attitude and attitude rate, using only the solar vector data from RAX-1. The flight data was recorded in late December 2010 and at this time RAX-1 was nominally spinning around its long $(z)$ axis. As RAX-1 carried additional attitude sensors, these can be used to assess the accuracy of attitude estimates. The full filter estimate included in Figure 12 is an independent attitude estimate generated by a traditional filter that uses all available sensor data, including the onboard gyros, and is used as a truth metric for the attitude of the RAX-1 spacecraft. The full filter is fully described in Ref. [10].

Figure 13 shows the attitude and attitude rate errors for the filter, defined as the difference between the MEKF and the full filter. Attitude errors on 
each axis are typically below $5^{\circ}$, but can rise above $20^{\circ}$ for short periods. The filter's $1 \sigma$ estimate of uncertainty, shown as the dashed red line, matches the actual errors well. This is important as it means that the filter's estimated uncertainty is an accurate representation of the actual error.
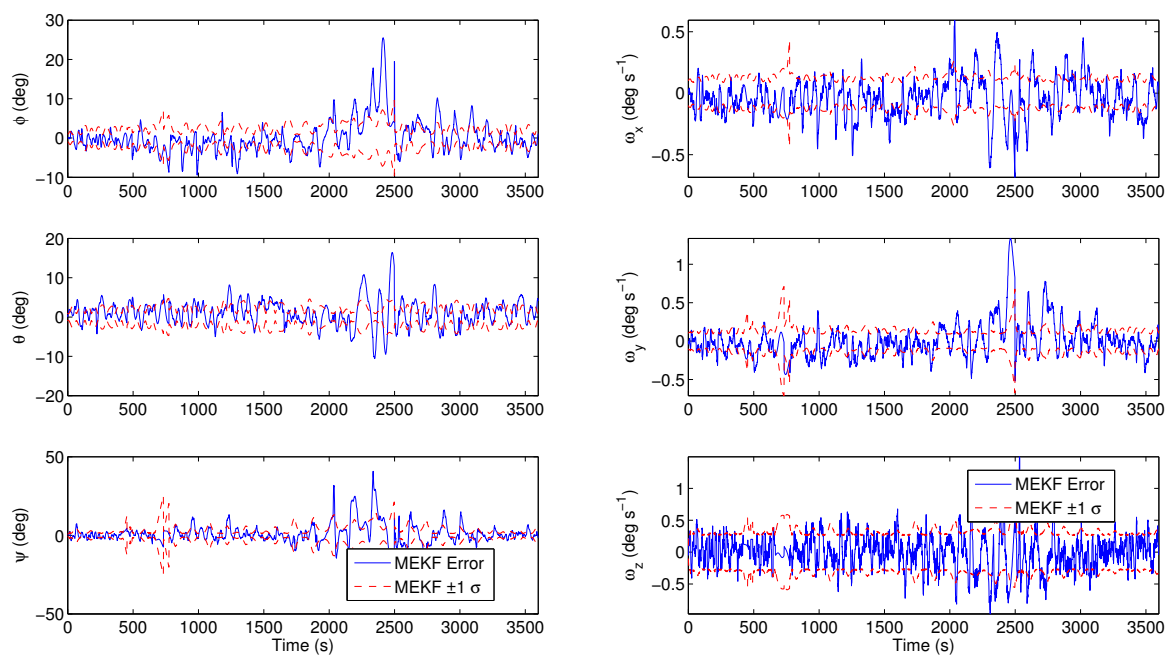

(a) Euler Angles, $(\phi, \theta, \psi)$

(b) angular velocity, $\omega$

Figure 13: Errors of the MEKF Attitude Filter for RAX-1. Plotted in blue is the error of the MEKF, defined as the difference between the full filter estimate and the MEKF estimate. Plotted in dashed red are the $\pm 1 \sigma$ bounds as determined by the MEKF. The errors in each Euler angle are around $4^{\circ}$, but with a divergence around $t=2000 \mathrm{~s}$, when errors grow to $20^{\circ}$ in $\phi, 10^{\circ}$ in $\theta$, and over $30^{\circ}$ in $\psi$. Errors in angular velocity are typically $0.2^{\circ} \mathrm{s}^{-1}$ in the $x$ and $y$ components and $0.4^{\circ} \mathrm{s}^{-1}$ in the $z$ component. Again some divergence is experienced, although starting at nearer $t=2500 \mathrm{~s}$. The observed deterioration in performance is explained by a combination of observability and Earth albedo.

Finally the overall attitude determination error is shown in Figure 14(a), Attitude determination accuracy is nominally $5^{\circ}$, but with periods of deteriorated performance.

\subsection{Observability and Albedo}

The poor filter performance for RAX-1 starting at around $t=2000 \mathrm{~s}$ can be partially explained by considering the geometry of the spacecraft and 


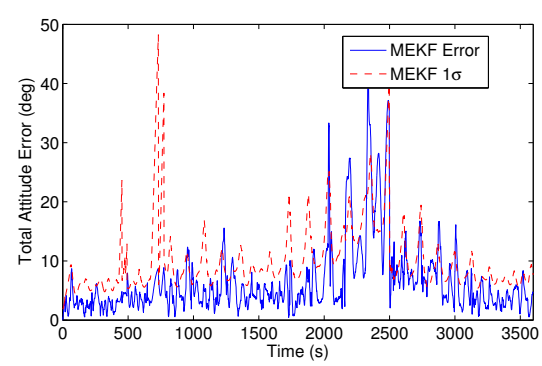

(a) MEKF Total Attitude Error

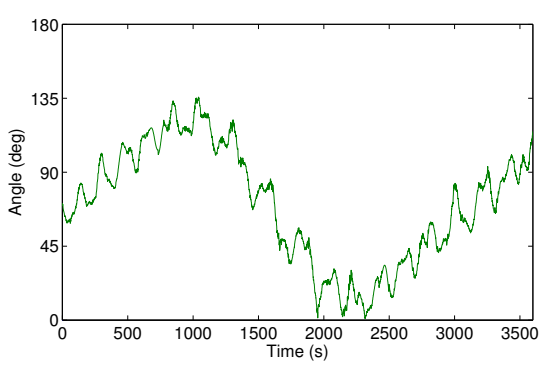

(b) Angle between spin vector and sun vector

Figure 14: RAX-1 Pointing Knowledge and Observability. Plotted in Figure 14(a) in blue is the total attitude error of the MEKF, defined as the angular difference between the full filter and the MEKF estimated attitude. Plotted in dashed red is the $1 \sigma$ bound of this estimate. Total attitude error is typically $5^{\circ}$, but grows to in excess of $40^{\circ}$ around $t=2200 \mathrm{~s}$. This growth in attitude error can be explained by a combination of observability and Earth albedo. Observability is effected by the angle between the sun vector and spin vector, as plotted in Figure 14(b). When the vectors become close to aligned, at around $t=2000 \mathrm{~s}$, performance is expected to deteriorate, and the filter's own uncertainty estimate (the $1 \sigma$ bound) predicts this reduction in performance well.

observations at that time, as was first noted in simulation in Section 5. Figure 14(b) plots the angle between the spin vector and the solar vector. When these two vectors are aligned, the spin rate is unobservable, as are angular deviations around the spin axis. The RAX-1 spacecraft has a permanent dipole that is close to $z$-aligned, but with a small $x$ component. Examining Figure 12(b) does show that the spin rate is closest to $z$-aligned but with a small $x$ component. It would be expected that during the period of poor observability the angular errors would be concentrated in the $x$ and $z$, or $\phi$ and $\psi$ axes, and Figure 13(a) confirms this observation.

During the period of poor observability total attitude errors approach $50^{\circ}$, much higher than the $25^{\circ}$ seen in simulation in Section 5 . One possible cause of the additional performance deterioration is suggested by examining the raw measurements coming from the photodiodes during that period. Figure 15(a) plots the raw photodiode readings during the time period in question, where the voltages from the sensors on the negative facing pan-

415 els have been inverted. As can be seen, starting around $t=2000 \mathrm{~s}$ some anomalous behavior occurs. Normally it would not be expected that both the positive and negative facing panels would be illuminated at the same 

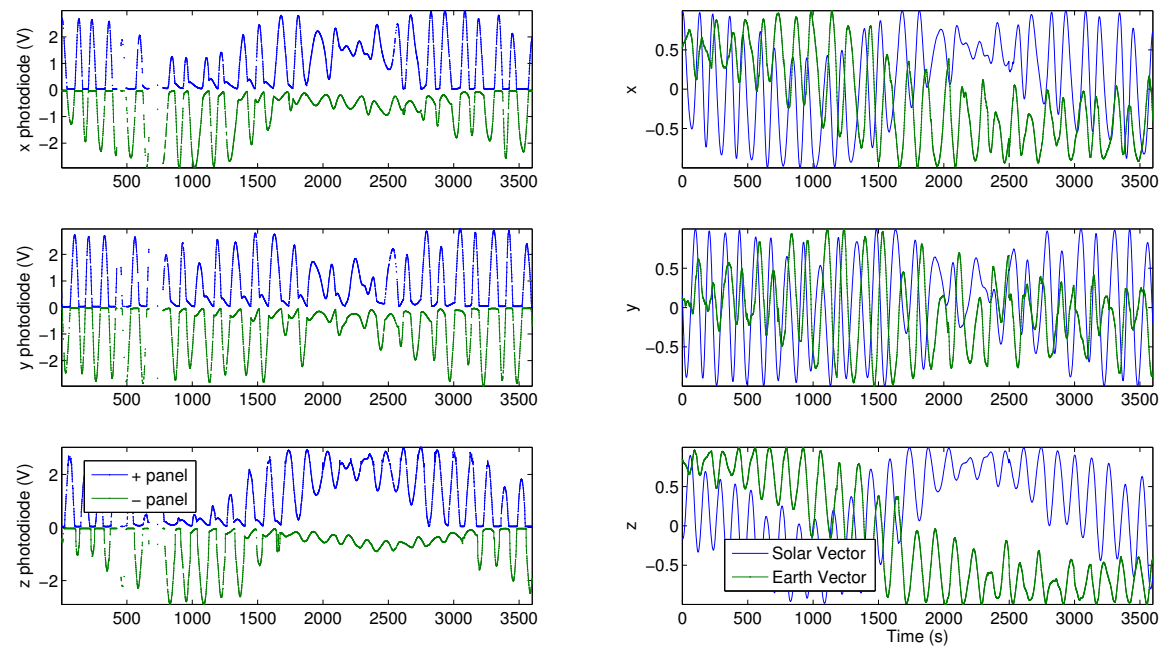

(a) Raw Solar Vector Readings

(b) Sun and Earth Unit Vectors

Figure 15: Impact of Earth Albedo on Solar Vector. In Figure 15(a), the raw measurements from the photodiode on the positive-facing panel are plotted in blue, and the negative of the raw measurements from the photodiode on the negative-facing panel are plotted in green, for the $\pm x, \pm y$ and $\pm z$ panels. The two raw measurements from opposing sensors are not expected to both be positive at the same time. However, starting at around $t=2000 \mathrm{~s}$, both the $+x$ and $-x$ and the $+z$ and $-z$ facing photodiodes have simultaneous readings. The smaller signals from the $-x$ and $-z$ facing photodiodes can be explained by Earth albedo. Figure 15(b) plots the Earth (green) and solar (blue) unit vectors in the spacecraft frame, showing that around $t=2000 \mathrm{~s}$, the $-x$ and $-z$ facing photodiodes would have a view of the Earth and would register sunlight reflected by the Earth.

time. As can be seen though, this occurs for the $\pm x$ and $\pm z$ facing panels between $t=2000 \mathrm{~s}$ and $t=2500 \mathrm{~s}$.

420 This anomalous behavior can be explained by the affect of Earth albedo illuminating the spacecraft. Figure 15(b) plots the unit vectors in the spacecraft frame to the sun and the Earth. For the period of interest, between $t=2000 \mathrm{~s}$ and $t=2500 \mathrm{~s}$, the Earth is located on the opposite side of the $x$ and $z$ panels to the sun, causing the negative facing panel to be illuminated. The raw solar reading preprocessing algorithm subtracts the small readings caused by albedo from the true readings caused by the sun, causing an error in the estimate of the solar vector.

Online attitude estimation accuracy could be improved by including an 
albedo model. However as this is an attitude dependent effect, it could not be included in the pre-processing of the raw solar data, rather the attitude estimation filter itself would have to include a model accounting for both sun and Earth position. The measurement equation would directly use the solar panel currents rather than an estimate of the solar vector.

Filter performance was observed to only be impacted by albedo when both the spin-axis and the solar vector are aligned and when the Earth and sun are in opposing quadrants. Outside of this scenario, performance impact was negligible. The additional complexity of a filter that includes a model for Earth albedo has to be balanced against the importance of attitude estimation accuracy for all possible geometries.

\subsection{Lost In Space Initialization}

The filter is capable of converging from a lost-in-space initialization. In such a case the initial estimate of the kinematic state would be the identity quaternion and zero angular velocities. A sample convergence is illustrated in Figure 16 and 17(a), showing convergence from an initial pointing error of over $150^{\circ}$. The lost in space scenario was simulated using real flight data with unknown initial conditions.

\subsection{Eclipse Performance}

Unsurprisingly the online filter is unable to provide good attitude estimates in eclipse. While in eclipse no measurements can be taken and filter accuracy is reliant on the accuracy of the state estimate just prior to entering eclipse. Given typical pointing knowledge errors of $5^{\circ}$ it is unlikely that the state estimate on entering eclipse will be good enough to maintain accuracy for the duration of the eclipse. As the filter is able to converge from a lostin-space initialization, it is not surprising that the MEKF is able to converge to the correct estimate once re-entering sunlight.

\section{Conclusions}

In this paper a filter was developed for performing online attitude estimation of a passively magnetically stabilized nano satellite with no dedicated attitude determination hardware installed. Only an estimate of the sun vector, determined from solar panel currents, was used. The filter was successfully tested using actual flight data from O/OREOS, a satellite that had no attitude sensors installed and provided the motivating problem for this work. 

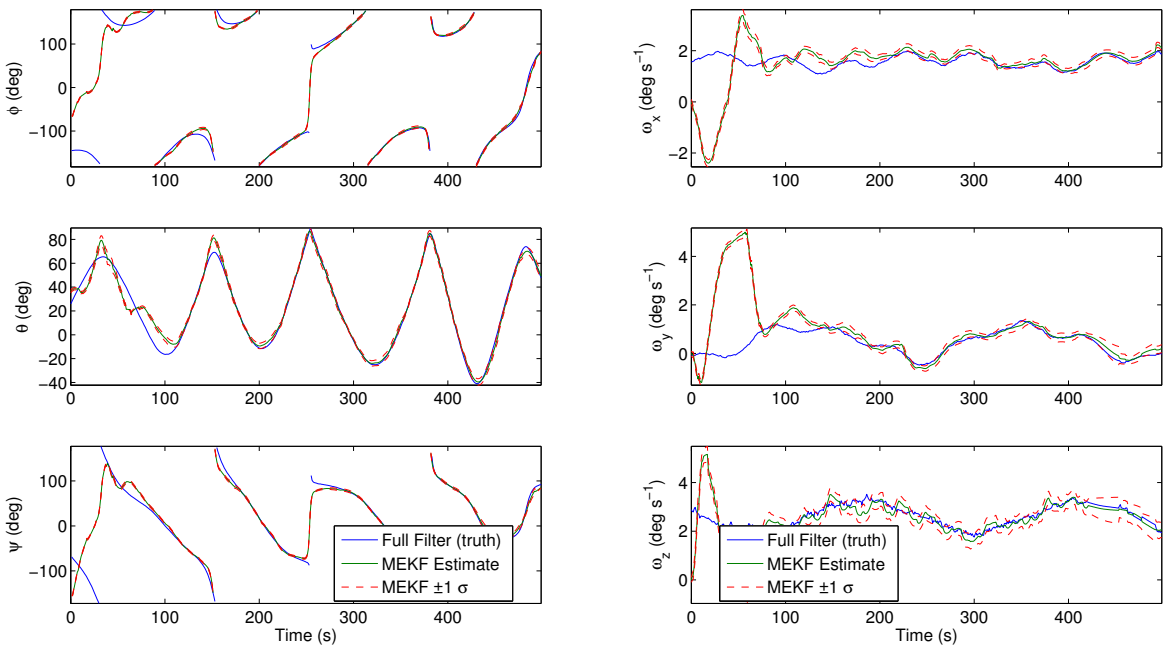

(a) Euler Angles, $(\phi, \theta, \psi)$

(b) angular velocity, $\omega$

Figure 16: Convergence of the MEKF Attitude Filter with Lost in Space Initialization for RAX-1. Plotted in blue is the full filter estimate (a truth metric), in green is the estimate of the MEKF filter and in dashed red the $\pm 1 \sigma$ bound as determined by the filter. The filter is observed to converge from a lost in space initialization to the correct attitude within a few minutes.

Filter performance was verified to an accuracy of around $5^{\circ}$ using actual flight data from RAX-1. The filter was a modification of the existing multiplicative extended Kalman filter (MEKF), replacing the gyro driven motion model with one derived from the rotational dynamics of the spacecraft and so removing the requirement to have onboard gyros. This modification requires the availability of an accurate model of the dynamics model.

\section{Acknowledgements}

The research described in this paper was funded by a grant from the National Aeronautics and Space Administration. Orbital data for the O/OREOS spacecraft was provided by Santa Clara University. Orbital data for the RAX-1 spacecraft was provided by the University of Michigan. RAX-1 was funded by the U.S. National Science Foundation 


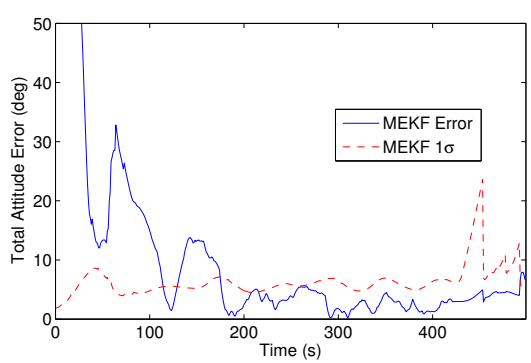

(a) Lost In Space Initialization

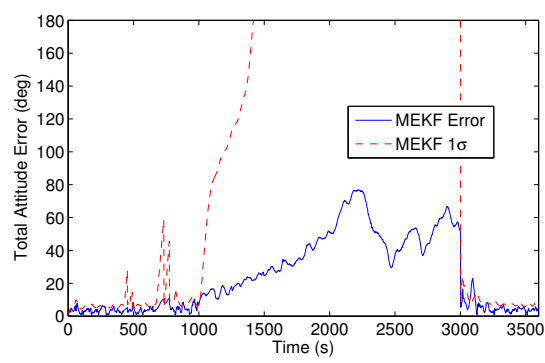

(b) Performance in Eclipse

Figure 17: MEKF Total Attitude Error for RAX-1. Plotted in blue is the total attitude error of the MEKF, defined as the angular difference between the full filter and the MEKF estimated attitude. Plotted in dashed red is the $1 \sigma$ bound of this estimate. From a lost in space initialization, the filter converges within a few minutes. During eclipse the MEKF is unable to maintain accuracy, and the attitude error grows to over $45^{\circ}$.

\section{Notation}

$\mu_{0} \quad$ permeability of free space $=4 \pi \times 10^{-7}$

$\mathrm{H} \mathrm{m}^{-1}$

c solar unit vector in body frame

$c_{\mathrm{ECI}}$ solar unit vector in inertial frame

$H \quad$ external magnetic field, body frame

$\mathrm{A} \mathrm{m}^{-1}$

$H_{\text {ECI }}$ Earth's magnetic field, ECI frame

$\mathrm{A} \mathrm{m}^{-1}$

$B \quad$ induced magnetic flux density in permeable material

$\mathrm{T}$

$V \quad$ volume of permeable rod material

$\mathrm{m}^{3}$

$M \quad$ total dipole of magnetic material

$\mathrm{A} \mathrm{m}^{2}$

$M_{\mathrm{P}} \quad$ permanent dipole in a permanent magnet

$\mathrm{A} \mathrm{m}^{2}$

$I$ moment of inertia, body frame

$\mathrm{kg} \mathrm{m}{ }^{2}$

$T_{\text {dist }}$ external disturbance torque, body frame

$\mathrm{Nm}$

$q \quad$ unit quaternion, inertial to body frame

a attitude error vector

$\omega$ angular velocity, body frame

$\operatorname{rads} s^{-1}$

$t \quad$ time

$\mathrm{S}$

$\Sigma \quad$ state covariance matrix

$\nu \quad$ measurement noise

$\eta \quad$ system noise

$\Sigma_{\nu} \quad$ measurement noise covariance matrix

$\Sigma_{\eta} \quad$ system noise covariance matrix

$\sigma_{a_{0}} \quad$ Standard deviation of uncertainty in initial attitude error

$\sigma_{\omega_{0}} \quad$ Standard deviation of uncertainty in initial attitude rate $\operatorname{rad~s}^{-1}$

$\sigma_{c} \quad$ Standard deviation of errors in solar vector

$\sigma_{H} \quad$ Standard deviation of errors 30 the external magnetic field $\mathrm{A} \mathrm{m}^{-1}$

$\sigma_{T} \quad$ Standard deviation of external disturbance torques $\quad \mathrm{A} \mathrm{m}^{-1}$ 
[1] P. Ehrenfreund, A. Ricco, D. Squires, C. Kitts, E. Agasid, N. Bramall, K. Bryson, J. Chittenden, C. Conley, A. Cook, R. Mancinelli, A. Mattioda, W. Nicholson, R. Quinn, O. Santos, G. Tahu, M. Voytek,

480 C. Beasley, L. Bica, M. Diaz-Aguado, C. Friedericks, M. Henschke, D. Landis, E. Luzzi, D. Ly, N. Mai, G. Minelli, M. McIntyre, M. Neumann, M. Parra, M. Piccini, R. Rasay, R. Ricks, A. Schooley, E. Stackpole, L. Timucin, B. Yost, A. Young, The o/oreos mission - astrobiology in low earth orbit, Acta Astronautica (0) (2012) -. doi: $10.1016 / \mathrm{j}$.actaastro.2012.09.009.

[2] J. Sedlak, D. Chu, Kalman filter estimation of attitude and gyro bias with the quest observation model, NASA STI/Recon Technical Report A 95 (1993) 85764.

[3] J. Crassidis, F. Markley, Y. Cheng, Survey of nonlinear attitude estimation methods, Journal of guidance control and dynamics 30 (1) (2007) 12 .

[4] F. L. Markley, Attitude error representations for kalman filtering, Journal of Guidance, Control, and Dynamics 26 (2) (2003) 311-317. doi: $10.2514 / 2.5048$.

[5] R. Burton, S. M. Rock, J. Springmann, J. Cutler, Dual attitude and parameter estimation of passively magnetically stabilized nano satellites, Acta Astronautica 94 (1) (2014) 145 - 158. doi:http://dx.doi.org/ $10.1016 /$ j.actaastro.2013.08.017.

[6] J. Cutler, J. Springmann, S. Spangelo, H. Bahcivan, Initial flight assessment of the radio aurora explorer, proceedings of the 25th Annual AIAA/USU Conference on Small Satellites. SSC11-VI-6. Logan, Utah.

[7] J. Springmann, A. Sloboda, A. Klesh, M. Bennett, J. Cutler, The attitude determination system of the rax satellite, Acta Astronautica 75 (2012) 120-135.

[8] J. Springmann, Attitude-independent magnetometer calibration with time-varying bias, Proceedings of the 25th Annual Small Satellite Conference.

[9] D. Vallado, P. Crawford, R. Hujsak, T. Kelso, Revisiting spacetrack report\# 3, AIAA 6753 (2006) 2006. 
510

520

[10] R. Burton, Attitude determination of passively magnetically stabilized nano satellites, Ph.D. thesis, Stanford University (May 2013).

[11] E. Lefferts, F. Markley, M. Shuster, Kalman filtering for spacecraft attitude estimation, Journal of Guidance, Control, and Dynamics 5 (5) (1982) 417-429.

[12] J. Taylor, The cramer-rao estimation error lower bound computation for deterministic nonlinear systems, in: Decision and Control including the 17th Symposium on Adaptive Processes, 1978 IEEE Conference on, Vol. 17, IEEE, 1978, pp. 1178-1181.

[13] International Association of Geomagnetism and Aeronomy, Working Group V-MOD. Participating members, C. C. Finlay, S. Maus, C. D. Beggan, T. N. Bondar, A. Chambodut, T. A. Chernova, A. Chulliat, V. P. Golovkov, B. Hamilton, M. Hamoudi, R. Holme, G. Hulot, W. Kuang, B. Langlais, V. Lesur, F. J. Lowes, H. Lühr, S. Macmillan, M. Mandea, S. McLean, C. Manoj, M. Menvielle, I. Michaelis, N. Olsen, J. Rauberg, M. Rother, T. J. Sabaka, A. Tangborn, L. TøffnerClausen, E. Thébault, A. W. P. Thomson, I. Wardinski, Z. Wei, T. I. Zvereva, International geomagnetic reference field: the eleventh generation, Geophysical Journal International 183 (3) (2010) 1216-1230. doi:10.1111/j.1365-246X.2010.04804.x.

[14] F. Santoni, M. Zelli, Passive magnetic attitude stabilization of the unisat-4 microsatellite, Acta Astronautica 65 (5-6) (2009) 792 - 803. doi:10.1016/j.actaastro.2009.03.012.

[15] R. Burton, J. Starek, S. M. Rock, A New Method for Simulating the Attitude Dynamics of Passively Magnetically Stabilized Spacecraft, in: Proceedings of the 22nd AAS/AIAA Space Flight Mechanics Meeting, AAS/AIAA, AAS/AIAA, Charleston SC, 2012. 